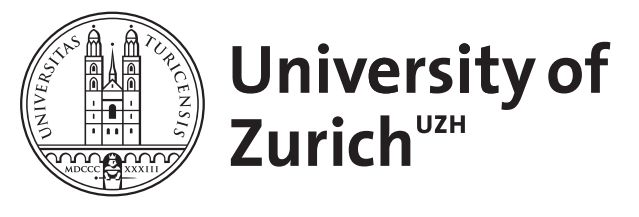

\title{
Diffuse Bauchschmerzen und Eosinophilie
}

\author{
Hochholzer, W ; Vavricka, S R ; Stussi, G ; Adams, H ; Wruk, D ; Battegay, E ; Zimmerli, L U
}

\begin{abstract}
Die eosinophile Gastroenteritis ist eine relativ seltene Erkrankung unklarer Ätiologie mit einem heterogenen Krankheitsbild. Wichtige Differenzialdiagnosen stellen intestinale parasitäre Infektionen, das hypereosinophile Syndrom, Lymphome und andere maligne und allergische Erkrankungen dar. Die Diagnose kann meist mittels Anamnese und Standardlabor, zusammen mit den Ergebnissen von Biopsien oder Parazentese gestellt werden. Milde Formen mit lediglich Diarrhö als klinischer Manifestation können symptombezogen behandelt werden. Die Therapie bei schwereren Verläufen erfolgt initial mit Kortikosteroiden. = Eosinophilic gastroenteritis is a rare clinical condition of unknown aetiology and heterogenic etiopathology. Important differential diagnoses are intestinal parasitic infections, hypereosinophilic syndrome, malignancies such as lymphoma and allergic diseases. The diagnosis can be made in most cases by patient history, routine laboratory testing and endoscopic biopsies or paracentesis. Patients with only mild diarrhea can be treated with antidiarrheal medications. More symptomatic patients are usually treated with corticosteroids.
\end{abstract}

DOI: https://doi.org/10.1007/s00108-008-2164-2

Other titles: Diffuse abdominal pain and eosinophilia

Posted at the Zurich Open Repository and Archive, University of Zurich

ZORA URL: https://doi.org/10.5167/uzh-14421

Journal Article

Published Version

Originally published at:

Hochholzer, W; Vavricka, S R; Stussi, G; Adams, H; Wruk, D; Battegay, E; Zimmerli, L U (2008). Diffuse Bauchschmerzen und Eosinophilie. Der Internist, 49(11):1379-1382.

DOI: https://doi.org/10.1007/s00108-008-2164-2 
Internist 2008 • 49:1379-1382

DOI 10.1007/s00108-008-2164-2

Online publiziert: 29. August 2008

(c) Springer Medizin Verlag 2008

\section{Rubrikherausgeber}

K. Werdan, Halle/Saale

W. Hochholzer ${ }^{1}$ S.R. Vavricka ${ }^{2} \cdot$ G. Stussi ${ }^{3,4} \cdot$ H. Adams $^{5}$ - D. Wruk ${ }^{6} \cdot$ E. Battegay $^{7} \cdot$ L.U. Zimmerli ${ }^{1,7}$

${ }^{1}$ Medizinische Poliklinik, Universitätsspital Basel

${ }^{2}$ Abteilung für Gastroenterologie und Hepatologie, Universitätsspital Basel

${ }^{3}$ Abteilung für Hämatologie, Universitätsspital Basel

${ }^{4}$ Klinik für Hämatologie, Universitätsspital Zürich

${ }^{5}$ Institut für Pathologie, Universitätsspital Basel

${ }^{6}$ Institut für Radiologie, Universitätsspital Basel

${ }^{7}$ Klinik und Poliklinik für Innere Medizin, Universitätsspital Zürich

\section{Diffuse Bauchschmerzen und Eosinophilie}

lichen Darmgeräuschen. Der Neurostatus war unauffällig ohne Meningismus, auch Haut und Schleimhäute waren nicht auffällig.

Die 43-jährige Patientin wurde im Frühjahr 2007 wegen seit einigen Tagen anhaltender, diffuser Bauchschmerzen von brennendem Charakter zur weiteren $\mathrm{Ab}$ klärung zugewiesen. Sie hatte bis zu 5-mal täglich breiig-wässrigen Stuhlgang ohne Blutbeimengungen. Zudem klagte sie über ein Völlegefühl und eine Zunahme des Bauchumfangs bei stabilem Gewicht. Sie hatte kein Fieber und keinen Schüttelfrost. Sie verneinte regelmäßige Medikamenteneinnahme, Alkohol-, Nikotinoder Drogenkonsum.

Die persönliche Anamnese war bis auf eine Allergie auf Gräser und Tierhaare unauffällig. Fünf Monate zuvor war die Patientin auf Safari in Kenia (Malariaprophylaxe mit Atovaquon/Proguanil). In der Familienanamnese verstarb ein Bruder 38jährig an einem abdominalen High-grade-Sarkom mit Peritonealkarzinose.

\section{Klinischer Befund}

Die afebrile 43-jährige Patientin zeigte sich in einem guten Allgemeinzustand bei einem Body Mass Index von 24,5 kg/ $\mathrm{m}^{2}$. Blutdruck (125/75 mmHg), Puls (82/ min), Herz und Lunge waren unauffällig. Das Abdomen war weich ohne Resistenzen, Klopf- oder Druckdolenzen bei spär-

\section{Diagnostik}

\section{Laboruntersuchungen}

Im Blutbild zeigte sich eine ausgeprägte Eosinophilie: Leukozyten $9,12 \times 10^{9} / 1$, Eosinophile 4,29 $\times 10^{9} / 1$ (Norm: $\left.\leq 0,7 \times 10^{9} / \mathrm{L}\right)$. CRP, die Basislaborwerte (Chemogramm) und Rheumalabor waren regelrecht, Gesamt-IgE mit >200 IU erhöht, Urinstatus und Sediment waren unauffällig. Die Stuhlkulturen waren negativ. Im tropenmedizinischen Labor (Serologien, Stuhl in
Fixationslösung und nativ) fand sich kein Hinweis auf eine Parasitose.

\section{Abdomensonographie und Aszitespunktion}

Sonographisch zeigte sich ein ausgeprägter Aszites, nach der Parazentese fand sich in der Analyse ein trübes Exsudat mit 1,1×10 $9 / 1$ Zellen, davon 57\% Eosinophile (- Abb. 1). Zytologisch fanden sich keine Hinweise auf Parasiten oder Malignität, in der Kultur kein Bakterienwachstum.

\section{Computertomographie}

Thorax und Abdomen/Becken. Thorakal bestand dorsobasal rechts ein Pleura-

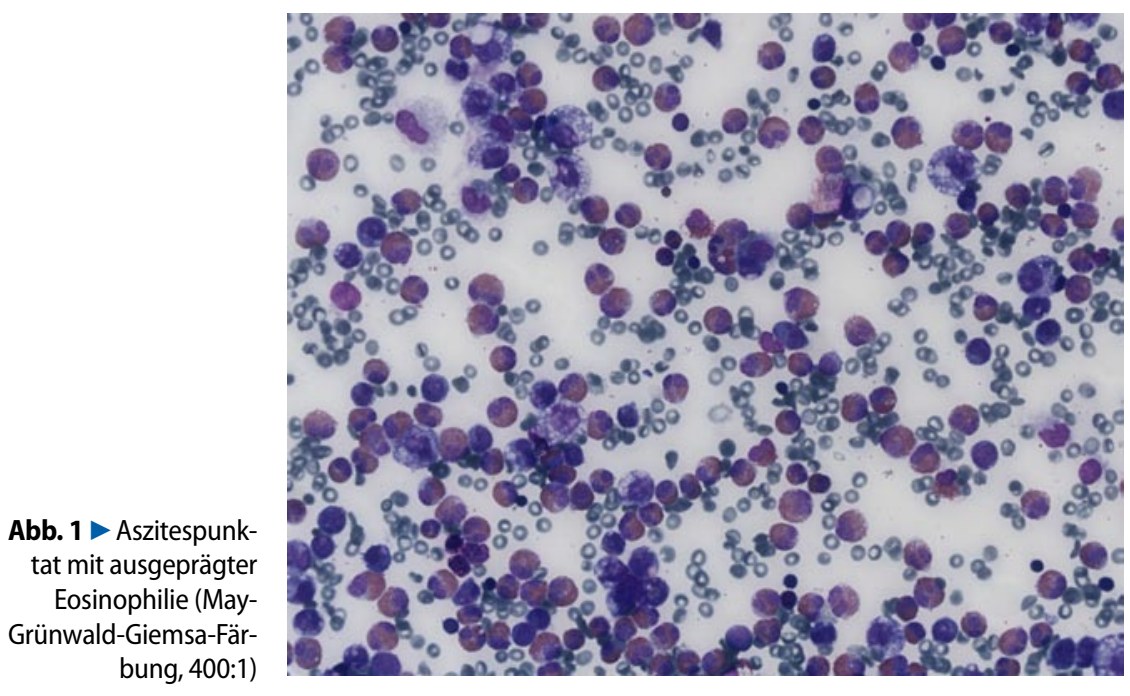




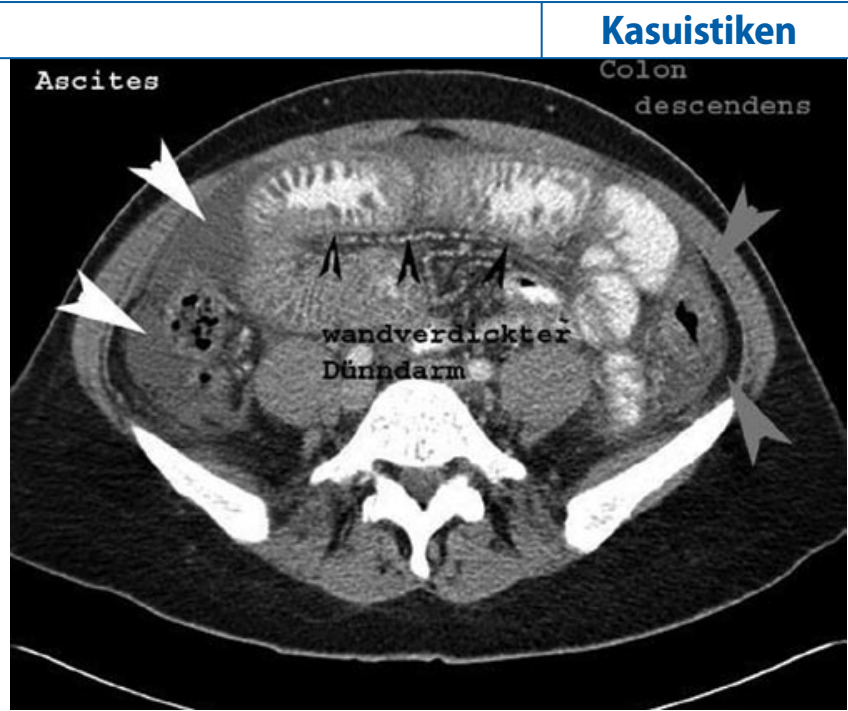

Abb. $2 \Delta$ Computertomographie des Abdomens mit ausgeprägter Kolitis (dunkle Pfeile) und Aszites (helle Pfeile)

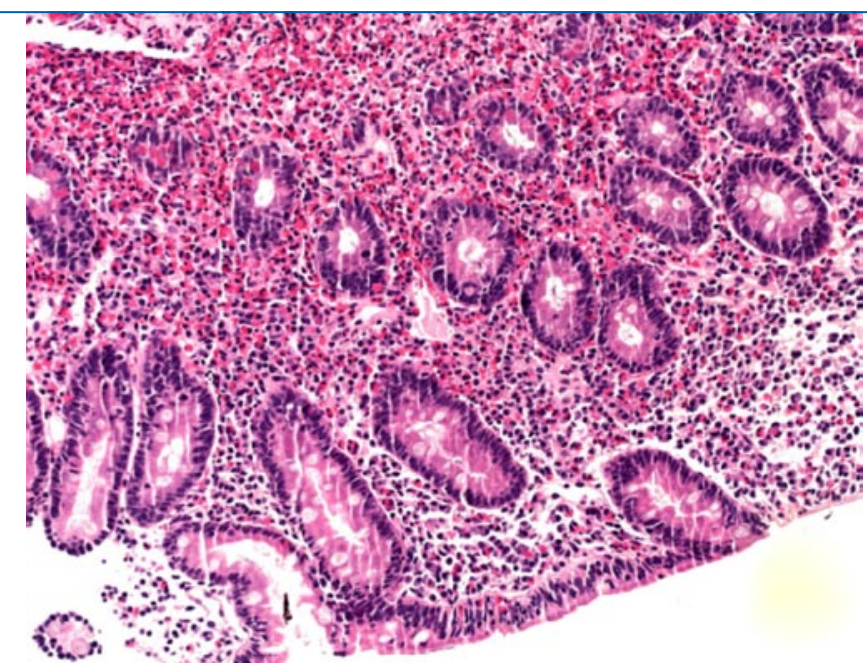

Abb. $3 \Delta$ Duodenalschleimhaut mit dichter Infiltration durch Eosinophile und vollständiger Zottenatrophie (Hämatoxylin-Eosin, 200:1)

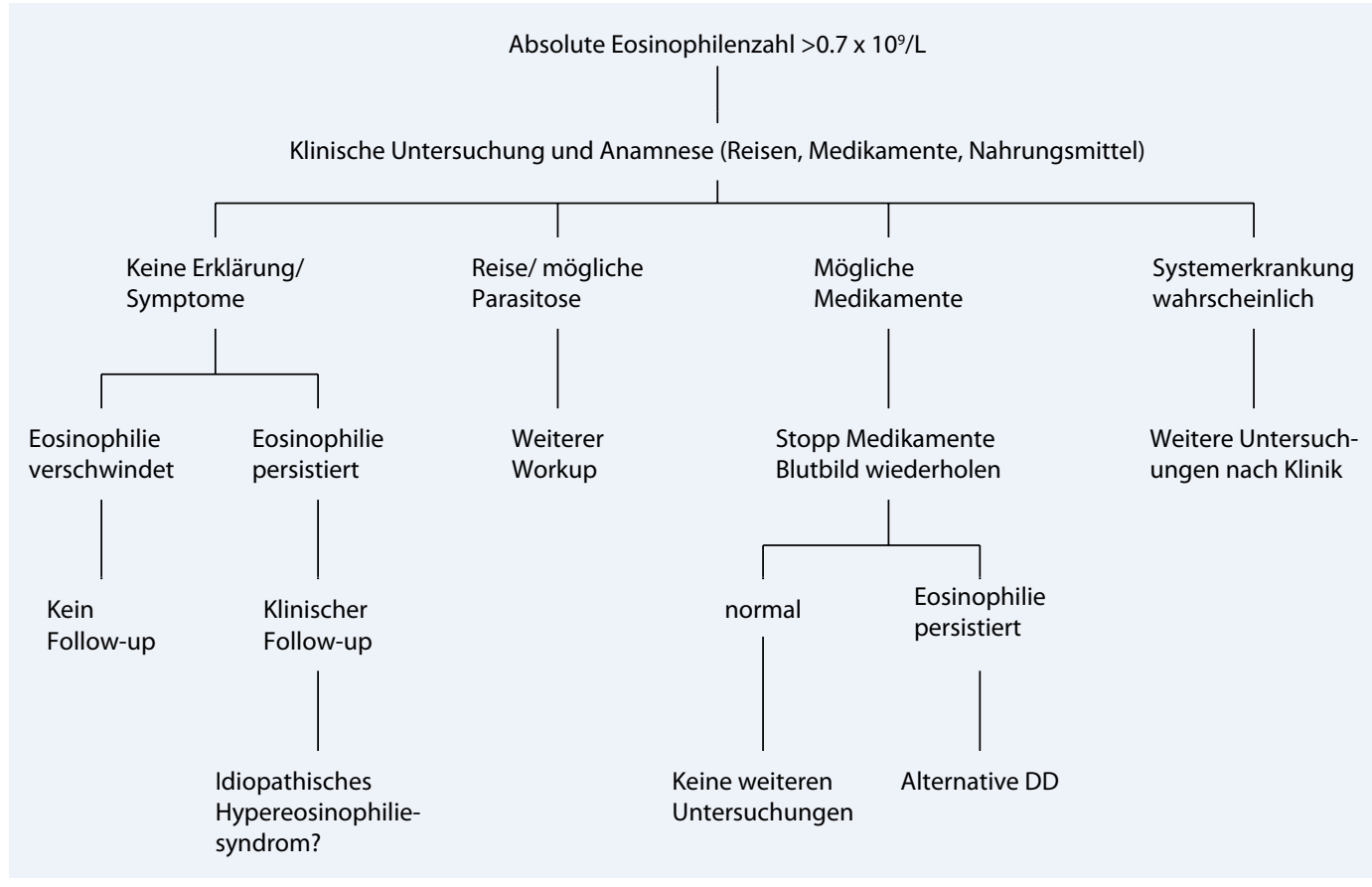

Abb. $4<$ Abklärungsalgorithmus für die Eosinophilie. (Mod. nach [3]) erguss, abdominal fanden sich ein ausgedehnter Aszites, eine ausgeprägte Kolitis ohne Beteiligung von Zäkum und Rektum sowie segmentale Wandverdickungen von Jejunum und Ileum (• Abb. 2).

Schädel. Diese zur weiteren Tumorsuche durchgeführte Untersuchung war unauffällig.

\section{Echokardiographie}

Die Echokardiographie wurde zum Ausschluss eines Organbefalls bei möglichem hypereosinophilem Syndrom durchgeführt. Die Untersuchung erbrachte einen altersentsprechenden Normalbefund.

\section{Gastro- und Koloskopie}

Bei der Gastroskopie zeigten sich makroskopisch keine pathologischen Befunde, histologisch fand sich im Duodenum eine vollständige Zottenatrophie mit ausgeprägter eosinophiler Entzündung (• Abb. 3).

Bei der Koloskopie wurde makroskopisch eine minimale Kolitis im Sigma beschrieben, die Histologie war unauffällig.

\section{Knochenmarkpunktion}

Histologisch zeigte sich ein leicht hyperzelluläres trilinear durchreifendes Knochenmark mit 40\%iger interstitieller Eosinophilie bei gesteigerter Eosinophilo- poese mit Auftreten auch von vermehrten eosinophilen Vorläufern. Die Zytogenetik war negativ für KIT-D816V-Mutation und FIP1L1-PDGFRA (Hypereosinophilensyndrom, Mastozytose), negativ für $J A K_{2}$ Mutation (myeloproliferatives Syndrom) und negativ für $B C R-A B L$ (chronisch myeloische Leukämie). Somit gab es keine Hinweise auf eine lymphoproliferative Erkrankung mit reaktiver Eosinophilie.

\section{Diagnose \\ - Idiopathische subseröse eosinophile Gastroenteritis mit eosinophilem As- zites.}




\section{Therapie und Verlauf}

Im weiteren Verlauf wurde die Patientin mit Prednison behandelt, initial $50 \mathrm{mg}$ täglich, welches in der Folge schrittweise reduziert wurde. Darunter sanken die Eosinophilen, die klinische Symptomatik besserte sich rasch. Nach Sistieren der Steroidmedikation war die Patientin in den folgenden 2 Monaten beschwerdefrei, ehe es während eines Mittelmeerurlaubs wieder zu abdominalen Beschwerden kam. Im Blutbild zeigte sich erneut eine Eosinophilie $\left(2,30 \times 10^{9} / 1\right)$, weshalb eine neuerliche Therapie mit Prednison begonnen wurde. Darunter kam es wiederum zu einer raschen Besserung der Symptome. Die Steroide werden nun über einen längeren Zeitraum stufenweise reduziert.

\section{Diskussion}

\section{Eosinophile Gastroenteritis: Inzidenz und Pathogenese}

Die eosinophile Gastroenteritis ist eine seltene Erkrankung mit heterogenem Krankheitsbild [5]: Genaue Zahlen über die Inzidenz der Erkrankung sind nicht bekannt, allerdings findet sie sich am häufigsten bei Personen zwischen dem 20. und 5o. Lebensjahr. Eine geschlechtsabhängige Verteilung scheint nicht zu bestehen [4].

Die genaue Pathogenese der Erkrankung ist bislang unbekannt. Jedoch weisen einige epidemiologische und klinische Beobachtungen auf eine allergische Komponente der Erkrankung hin. So haben etwa die Hälfte der Patienten andere allergische Erkrankungen wie Asthma bronchiale oder Nahrungsmittelallergien [10, 11]. Auch wurden bei einigen Patienten erhöhte IgE-Werte beschrieben $[1,5]$. Bislang konnte aber kein typischer Auslöser in der Nahrung identifiziert werden - auch das Vermeiden von individuellen Allergenen bei betroffenen Patienten führte zu keiner signifikanten Verbesserung der Symptomatik [5].

\section{Symptome}

Verschiedene Wandschichten des MagenDarm-Trakts können betroffen sein, die im Einzelfall zu unterschiedlichen Symp- tomen führen. Man unterscheidet daher zwischen einer Mukosa-, Muscularispropria- und Subserosa-Form $[2,5,10]$ :

Bei der Mukosa-Form stehen eher unspezifische Symptome im Vordergrund $[2,10]$, häufig werden Bauchschmerzen, Nausea und Diarrhö beklagt. Nur etwa ein Drittel der Patienten weist einen deutlichen Gewichtsverlust auf. Als Folge einer Malabsorption kann es zu einer Vitamin-K-abhängigen Verlängerung der Prothrombinzeit, zu Fettstühlen und einem Eisenmangel kommen. Eine Hypalbuminämie kann Folge eines enteralen Eiweißverlusts sein, eine Anämie durch enterale Mikroblutungen verursacht werden. Die Eosinophilie im Blutbild ist richtungweisend, in etwa 20\% der Fälle aber nicht vorhanden [10].

Bei der Muskularis-Form stehen Symptome der gastrointestinalen Obstruktion wie Nausea, Emesis und Spannungsschmerz im Vordergrund [2, 10]. Am häufigsten treten diese Veränderungen im oberen Gastrointestinaltrakt auf und können in Röntgenkontrastaufnahmen wie durch maligne Erkrankungen induzierte Strikturen imponieren.

Die Subserosa-Form ist gekennzeichnet durch das Auftreten von Aszites [10]. In der Punktion zeigt sich typischerweise eine ausgeprägte Eosinophilie. Da es auch parallel einen Befall der Mukosa und/ oder Muscularis propria geben kann, ist z. T. auch die Symptomatik dieser Formen vorhanden.

\section{Diagnose und Differenzialdiagnose}

Die Diagnose wird durch eine bestätigende Histologie respektive Zytologie gestellt. Andere Ursachen der Eosinophilie müssen ausgeschlossen werden. Wichtig ist, dass mehrere Biopsien entnommen werden, auch von gesund erscheinendem Gewebe.

\section{$>$ Eine eosinophile Gastroenteritis sollte vor der Diagnosestellung eines Reizdarmsyndroms in Betracht gezogen werden}

Bei Patienten mit gastrointestinalen Beschwerden und Eosinophilie sollte differenzialdiagnostisch an eine eosinophi-
Internist 2008 · 49:1379-1382

DOI 10.1007/s00108-008-2164-2

○) Springer Medizin Verlag 2008

W. Hochholzer · S.R. Vavricka · G. Stussi · H. Adams - D. Wruk · E. Battegay - L.U. Zimmerli

Diffuse Bauchschmerzen und Eosinophilie

\section{Zusammenfassung}

Die eosinophile Gastroenteritis ist eine relativ seltene Erkrankung unklarer Ätiologie mit einem heterogenen Krankheitsbild. Wichtige Differenzialdiagnosen stellen intestinale parasitäre Infektionen, das hypereosinophile Syndrom, Lymphome und andere maligne und allergische Erkrankungen dar. Die Diagnose kann meist mittels Anamnese und Standardlabor, zusammen mit den Ergebnissen von Biopsien oder Parazentese gestellt werden. Milde Formen mit lediglich Diarrhö als klinischer Manifestation können symptombezogen behandelt werden. Die Therapie bei schwereren Verläufen erfolgt initial mit Kortikosteroiden.

Schlüsselwörter

Eosinophilie · Gastroenteritis · Eosinophile Gastroenteritis · Aszites · Diarrhö

\section{Diffuse abdominal pain and eosinophilia}

\section{Abstract}

Eosinophilic gastroenteritis is a rare clinical condition of unknown aetiology and heterogenic etiopathology. Important differential diagnoses are intestinal parasitic infections, hypereosinophilic syndrome, malignancies such as lymphoma and allergic diseases. The diagnosis can be made in most cases by patient history, routine laboratory testing and endoscopic biopsies or paracentesis. Patients with only mild diarrhea can be treated with antidiarrheal medications. More symptomatic patients are usually treated with corticosteroids.

\section{Keywords}

Eosinophilia · Gastroenteritis · Eosinophilic gastroenteritis - Ascites · Diarrhea 


\section{Hier steht eine Anzeige.}

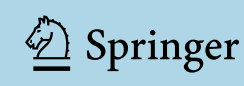

le Gastroenteritis gedacht werden. Da sie gut behandelt werden kann, sollte diese Möglichkeit auch vor der Diagnosestellung eines Reizdarmsyndroms in Betracht gezogen werden.

Mögliche Differenzialdiagnosen, welche alle mit einer Eosinophilie im Blutbild und gastrointestinalen Beschwerden einhergehen können, sind die allergische Gastroenteritis, intestinale Parasitosen (z. B. Strongyloiden, Trichinellen, Askarien), das hypereosinophile Syndrom, Lymphome, Magen- oder Kolontumore, chronisch entzündliche Darmerkrankungen (M. Crohn und Colitis ulcerosa), das Eosinophilie-Myalgie-Syndrom nach Einnahme von L-Tryptophan und auch Vaskulitiden, insbesondere das Churg-StraussSyndrom. Ein Abklärungsalgorithmus für die Eosinophilie findet sich in $\bullet$ Abb. 4.

\section{Therapie}

Ohne Behandlung sind sowohl Spontanheilungen als auch progrediente Verläufe, bis hin zur schweren Malabsorbtion, beobachtet worden [5, 7]. Diätetische Maßnahmen zeigen keinen nennenswerten Erfolg. Bei leichten Verläufen mit geringer Diarrhö hat sich eine Therapie mit einem Antidiarrhoikum (z. B. Loperamid) bewährt. Patienten mit ausgeprägten Symptomen der intestinalen Obstruktion, Hinweisen auf eine Malabsorption oder Aszites sollten initial mit Kortikosteroiden behandelt werden [2, 6]. Die Klinik bessert sich darunter meist innerhalb weniger Wochen.

Nach Beschwerdebesserung können die Steroide meist rasch ausgeschlichen werden. Der weitere Verlauf ist sehr variabel - einige Patienten bleiben nach einem Schub beschwerdefrei, während andere erneut Symptome entwickeln und z. T. eine Langzeittherapie mit Steroiden benötigen [5]. Größere Fallserien zu anderen immunsuppressiven Therapien (z. B. Azathioprin) liegen nicht vor. In Fallberichten wird von positiven Effekten durch Mastzellstabilisatoren und Leukotrienantagonisten berichtet $[8,9]$.

\section{Fazit für die Praxis}

Die eosinophile Gastroenteritis stellt zwar eine seltene Erkrankung dar. Sie sollte aber als mögliche Differenzialdi- agnose bei allen Patienten mit einer Eosinophilie im Blutbild und gastrointestinalen Symptomen in Erwägung gezogen werden, da sie meist gut behandelbar ist.

\section{Korrespondenzadresse}

Dr. L.U. Zimmerli

Klinik und Poliklinik für Innere Medizin,

Universitätsspital Zürich

Rämistrasse 100, 8091 Zürich

Schweiz

lukas.zimmerli@usz.ch

Interessenkonflikt. Der korrespondierende Autor gibt an, dass kein Interessenkonflikt besteht.

\section{Literatur}

1. Caldwell JH, Tennenbaum Jl, Bronstein HA (1975) Serum IgE in eosinophilic gastroenteritis. Response to intestinal challenge in two cases. $\mathrm{N}$ Engl J Med 292: 1388-1390

2. Cello JP (1979) Eosinophilic gastroenteritis - a complex disease entity. Am J Med 67: 1097-1104

3. Ebnoether M, Schoenenberger R (2005) Eosinophilie - was kommt in Frage? Schweiz Med Forum 5: 735-741

4. Fenoglio LM, Benedetti V, Rossi C et al. (2003) Eosinophilic gastroenteritis with ascites: a case report and review of the literature. Dig Dis Sci 48: 10131020

5. Klein NC, Hargrove RL, Sleisenger MH, Jeffries GH (1970) Eosinophilic gastroenteritis. Medicine (Baltimore) 49: 299-319

6. Lee CM, Changchien CS, Chen PC et al. (1993) Eosinophilic gastroenteritis: 10 years experience. Am J Gastroenterol 88: 70-74

7. Lee M, Hodges WG, Huggins TL, Lee EL (1996) Eosinophilic gastroenteritis. South Med J 89: 189-194

8. Melamed I, Feanny SJ, Sherman PM, Roifman CM (1991) Benefit of ketotifen in patients with eosinophilic gastroenteritis. Am J Med 90: 310-314

9. Quack I, Sellin L, Buchner NJ et al. (2005) Eosinophilic gastroenteritis in a young girl-long term remission under Montelukast. BMC Gastroenterol 5: 24

10. Talley NJ, Shorter RG, Phillips SF, Zinsmeister AR (1990) Eosinophilic gastroenteritis: a clinicopathological study of patients with disease of the mucosa, muscle layer, and subserosal tissues. Gut 31 : 54-58

11. Yun MY, Cho YU, Park IS et al. (2007) Eosinophilic gastroenteritis presenting as small bowel obstruction: a case report and review of the literature. World J Gastroenterol 13: 1758-1760 